\title{
Challenges in Differential Diagnosis: A Case Series of Four Adult Patients with Renal Leiomyoma
}

\author{
(1) Mehmet Necmettin Mercimek¹, • Ender Özden², (0 Yarkın Kamil Yakupoğlu² \\ 1 Samsun Liv Hospital, Clinic of Urology, Samsun, Turkey \\ 2 Ondokuz Mayıs University Faculty of Medicine, Department of Urology, Samsun, Turkey
}

\begin{abstract}
Leiomyomas are rare, benign and solid tumors of the kidney. Although the developments in radiological imaging methods provide early detection of kidney tumors, it is difficult to differentiate leiomyomas radiologically from other malignant renal tumors. Moreover, the definitive diagnosis of leiomyomas can only be achieved by histopathological and also immunohistochemical evaluation after surgical intervention. Immunohistochemically, positive staining with smooth muscle actin and vimentin, whereas negative staining with cytokeratin, S100, Mart1 and HMB45 are the methods used in the differential diagnosis of leiomyomas.

In this case series, after preoperative radiological evaluation, two female and two male patients between 45 and 89 years of age underwent laparoscopic treatment with the diagnosis of a malignant mass in the kidney. We aimed to illustrate the clinical, radiological and histopathological relationship of four adult patients who were managed by laparoscopic approach and diagnosed with renal leiomyoma. Renal leiomyomas should be kept in mind in patients with a renal mass before definitive treatment.
\end{abstract}

Keywords: Benign renal tumor, laparoscopy, radical nephrectomy, partial nephrectomy, renal leiomyoma

\section{Introduction}

Leiomyomas are infrequent solid, benign lesions of the kidney which arises from smooth muscle cells of various structures of kidney such as renal capsule, pelvis or vascular smooth muscle (1). In spite of their rarity within the genitourinary system, leiomyomas are frequently detected in the kidneys and are less often non-epithelial benign tumors of the bladder (2). Kidney leiomyomas are often identified between the second and fifth decade of life, with a median age of 42 years and a female predominance. Although the majority of kidney leiomyomas in the literature are adults, they can also be detected in children. Symptoms of leiomyomas vary according to localization in the urinary system, whereas those originating from the kidney often do not cause symptoms and may radiologically mimic other malignant masses of the kidney $(3,4)$.

The differential diagnosis can be difficult with clinical evaluation. Therefore, a variety of immunohistochemical staining methods such as cytokeratin, S100, Mart1 and HMB45 are needed for the definitive diagnosis of leiomyoma (1).

In this article, we aimed to discuss the rarely seen cases of renal leiomyomas from clinical evaluation to the definitive diagnosis in terms of clinical, radiological evaluation, surgical management, and histopathological and immunohistological assessment.

\section{Case Reports}

This study was conducted in accordance with the declaration of Helsinki. All patients read the patient information form and written informed consents were obtained.

Case 1: A 45-year-old woman with a history of open uterine myomectomy five years ago presented with pain in the left lower quadrant for one week. Physical examination, urine and blood analyses were normal. Abdominal ultrasonography (US) revealed a $5 \mathrm{~cm}$ mass in the lower pole of the right pelvic ptotic kidney. Further imaging with computerized tomography (CT) was performed and revealed a completely exophytic, wellcircumscribed, homogeneously contrast enhancing, $6 \times 5 \mathrm{~cm}$ renal mass (Figure $1 \mathrm{~A}$ and Figure 2). Since renal cell carcinoma could not be ruled out, laparoscopic partial nephrectomy was carried out for the management of the renal mass. Postoperative course was uneventful and the patient was discharged on the $3^{\text {rd }}$ post-operative day. Pathological gross examination of the surgical specimen revealed a $7 \times 5 \times 4 \mathrm{~cm}$ fibrillary mass in the form of a benign smooth muscle tumor with a hard-rubbery

Cite this article as: Mercimek MN, Özden E, Yakupoğlu YK. Challenges in Differential Diagnosis: A Case Series of Four Adult Patients with Renal Leiomyoma. Bull Urooncol 2019;18(4):158-161 
appearance surrounded by a thin fibrous capsule. A positive immunohistochemical staining with SMA (smooth muscle actin) also confirmed the pathologic diagnosis leiomyoma. Preoperative serum creatinine and eGFR were $0.5 \mathrm{mg} / \mathrm{dL}$ and $141.80 \mathrm{~mL} / \mathrm{min} / 1.73 \mathrm{~m}^{2}$, respectively. The patient was followed up for 11 months with a serum creatinine and eGFR of $0.6 \mathrm{mg} /$ $\mathrm{dL}$ and $114.90 \mathrm{~mL} / \mathrm{min} / 1.73 \mathrm{~m}^{2}$, respectively, at the last followup visit.

Case 2: On US examination, a 58-year-old man with microscopic hematuria was found to have a large mass located in the mid-lower pole of the right kidney. The patient had no additional systemic disease. Contrast-enhanced CT scan revealed an encapsulated right renal mass measuring $80 \times 80 \times 70 \mathrm{~mm}$ that medially pushed the renal pelvis and extended downward in the medial part of the right kidney. After contrast agent administration, homogeneous enhancement was observed in the central part of the mass with neovascularization in the inferior aspect of the mass, as well as peripheral heterogeneous enhancement. The remaining abdominal structures and left kidney had normal appearance, and no pathological lymph nodes were visualized. In the light of these radiological findings, the patient underwent a laparoscopic right radical nephrectomy. Histopathological examination of the mass revealed a leiomyoma that was uniformly separated from renal parenchyma, encapsulated, without mitosis, and immunohistochemically positive for SMA. The postoperative course was uneventful and the patient was discharged on the $3^{\text {rd }}$ postoperative day. Preoperative serum creatinine and eGFR were $0.9 \mathrm{mg} / \mathrm{dL}$ and $86 \mathrm{~mL} / \mathrm{min} / 1.73$ $\mathrm{m}^{2}$, respectively. The patient was followed up for 67 months. Serum creatinine and eGFR levels were $1.4 \mathrm{mg} / \mathrm{dL}$ and $58 \mathrm{~mL} /$ $\min / 1.73 \mathrm{~m}^{2}$, respectively, five years after surgery.

Case 3: A 4-cm middle pole mass was found on US in the right kidney of a 62-year-old man who was evaluated for lower urinary tract symptoms. The patient had no additional disease except for Type II diabetes mellitus that was regulated by oral antidiabetic. Two separate masses were detected on CT scan, one at the level of renal hilum, $38 \times 30 \mathrm{~mm}$ in size, located medially and the small one was $10 \times 7 \mathrm{~mm}$ in size, located anterolaterally in the right kidney (Figure 1B). Both masses had contrast-enhancement and a solid appearance. Laparoscopic right radical nephrectomy was carried out and histopathological evaluation was reported as leiomyoma for both masses. Immunohistochemical examination revealed negative staining with cytokeratin, CD10, S100 and positive staining with SMA and vimentin. Post-operative course was uneventful and the patient was discharged on the $2^{\text {nd }}$ post-operative day.

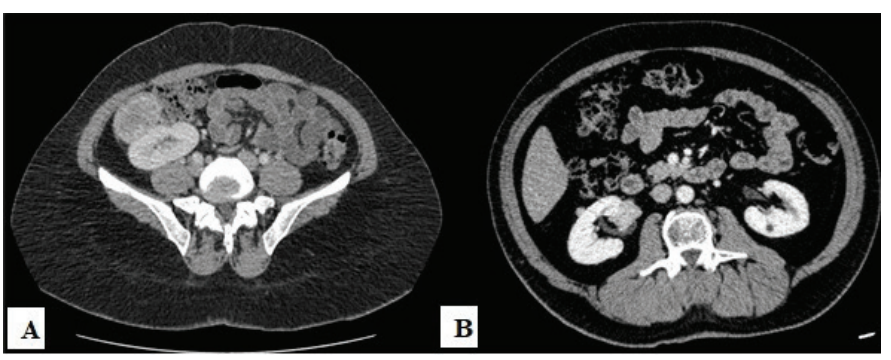

Figure 1. Contrast-enhanced CT images of Case 1 (A) and Case 3 (B) CT: Computerized tomography
Preoperative serum creatinine and eGFR were $0.7 \mathrm{mg} / \mathrm{dL}$ and $101 \mathrm{~mL} / \mathrm{min} / 1.73 \mathrm{~m}^{2}$, respectively. The patient was followed-up for 47 months with a serum creatinine and eGFR levels of 1.6 $\mathrm{mg} / \mathrm{dL}$ and $45 \mathrm{~mL} / \mathrm{min} / 1.73 \mathrm{~m}^{2}$, respectively.

Case 4: An 89-year-old woman who was evaluated for dyspeptic complaints was found to have two solid masses in her right kidney on abdominal US. Therefore, abdominal magnetic resonance imaging (MRI) was performed to obtain additional information about radiological characteristics of the masses. One $4 \mathrm{~cm}$ exophytic mass in the upper pole of the right kidney and one $3 \mathrm{~cm}$ solid mass in the medial cortex of the middle pole were detected. Moreover, the lesions were isointense on T1W images and hypo-intense on T2W images. The lesions were found to restrict diffusion on diffusion-weighted series. On dynamic multiphasic series, masses had more hypo-intense appearance and less contrast enhancement than the renal cortex. In the light of these findings, laparoscopic right radical nephrectomy was performed. Histopathological evaluation of the surgical specimen was reported as leiomyoma for both masses and immunohistochemical examination revealed negative staining with Mart1, HMB45 and positive staining with SMA. The postoperative course was uneventful and the patient was discharged on the $3^{\text {rd }}$ postoperative. The patient was followed-up for 14 months and, serum creatinine and eGFR levels were $1.12 \mathrm{mg} / \mathrm{dL}$ and $44 \mathrm{~mL} / \mathrm{min} / 1.73 \mathrm{~m}^{2}$, respectively.

\section{Discussion}

Leiomyomas may originate from smooth muscle cells of the renal capsule, renal pelvis and renal vascular structures of the upper urinary tract. Besides, it may also arise from other

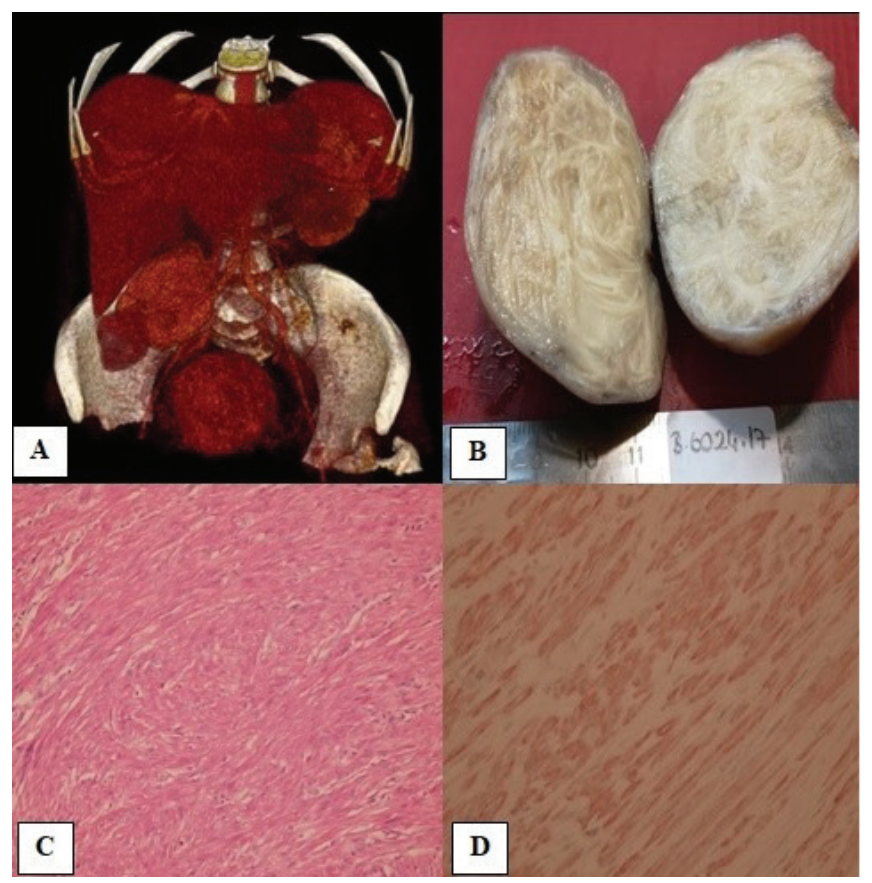

Figure 2. 3D-CT images of right pelvic kidney with mass (A) Macroscopic appearance of the lesion (B) Hematoxylin \& eosin staining $x 400$ (C) SMA staining $\times 400$ (D)

CT: Computerized tomography, SMA: Smooth muscle actin 
genitourinary tract organs including smooth muscle cells, such as ureter, bladder, urethra, prostate, seminal vesicles, testicles, penis and scrotum (5). Renal leiomyomas are extremely rare benign tumors of the kidney with a prevalence of up to $5 \%$, based on incidental findings of autopsy series. Renal leiomyomas constitute $1.5 \%$ of benign and $0.3 \%$ of overall renal tumors (6).

Although the vast majority of kidney leiomyoma cases reported in the literature are adults, they may also be detected in childhood. Leiomyomas are two times more common in women than men, and the incidence increases after the fourth decade of life $(3,5)$. In our series, leiomyoma was detected equally in both men and women. The cases were older than 40 years old and ranged between $45-89$ years.

Renal leiomyomas are divided into three groups in terms of location: a) subcapsular (53\%): small, multifocal, asymptomatic, incidentally detected tumors during autopsy or imaging, b) capsular (47\%): large, solitary and symptomatic masses (37\%), and c) rare renal pelvis leiomyomas (10\%) (4). Renal leiomyomas are detected incidentally on imaging as nonsymptomatic masses. However, when they reach large sizes and create mass effect, the common presenting symptoms are palpable flank mass, flank pain and hematuria. It was pointed out that symptomatic masses were seen more frequently in young patients and asymptomatic ones more frequently in the elderly (5). In our series, none of the four patients had palpable mass and flank pain, but one patient had microscopic hematuria and a large renal mass was detected incidentally in the right kidney.

In recent years, renal tumors are being detected at increasingly smaller sizes with the developments in radiological imaging. US is the first-line diagnostic tool if there is any solid or cystic renal masses. Contrast-enhanced CT and MRI provide detailed information about unidentified renal masses. However, radiologically, it is difficult to differentiate leiomyomas from other malign renal masses. In abdominal CT, small, peripherally located leiomyomas are seen as well-defined, capsulated, homogeneously enhancing solid lesions. But, it is stated that lesions show a lower enhancement than renal cortex at the corticomedullary phase, and also a cleavage plane between the renal cortex and lesion can be defined. As tumor size increases, the contrast pattern also shows heterogeneity due to hemorrhage, cystic or myxoid degeneration $(3,7)$. Besides, detailed preoperative anatomical information can be obtained by 3D-CT angiography for a renal mass with planned partial nephrectomy (8). In addition to the enhancement properties of leiomyomas, restricted diffusion may be detected in MRI due to different cellular and fibrous component contents (9).
The radiological findings of our cases were consistent with the literature. Since the right kidney is within the pelvic localization in Case 1, 3D-CT was performed for detailed anatomical information before laparoscopic partial nephrectomy.

In the light of current literature, the precise diagnosis of renal leiomyomas can be made after pathological examination. The cases described here could not be diagnosed preoperatively. Moreover, special immunohistochemical staining may be required for pathological diagnosis. Histopathologic differential diagnosis is determined according to staining characteristics with several stains such as SMA, vimentin, CD10, S100 and HMB45 (4).

Due to the difficulties of differential diagnosis with other malignant renal lesions, surgical removal is usually performed for benign kidney lesions such as leiomyomas. Open surgical methods have been preferred for patients with large renal masses in previous case reports. In recent years, minimally invasive methods such as laparoscopic or robot-assisted laparoscopic partial or radical nephrectomy have been preferred as surgical methods in appropriate patients $(10,11)$. To the best of our knowledge, this case series is the largest series in the literature in which renal leiomyomas were managed by laparoscopic methods. The characteristics of the cases are summarized in Table 1.

\section{Conclusion}

Today, despite the advancement in radiological diagnostic methods, it is difficult to differentiate renal leiomyomas from other malignant lesions in the preoperative period. Detailed pathological and immunohistochemical methods are needed for definitive diagnosis after surgical treatment. But, it is noticed that renal masses can be detected earlier in recent years. Therefore, the choice of minimally invasive treatment modalities, including partial nephrectomy, reduces patient morbidity and even mortality in long-term follow-up in appropriate patients.

\section{Ethics}

Informed Consent: All patients read the patient information form and written informed consents were obtained.

Peer-review: Externally peer-reviewed.

\section{Authorship Contributions}

Concept: M.N.M., E.Ö., Design: M.N.M., E.Ö., Data Collection or Processing: M.N.M., E.Ö., Y.K.Y., Analysis or Interpretation: M.N.M., E.Ö., Y.K.Y., Literature Search: M.N.M., E.Ö., Writing: M.N.M., E.Ö., Y.K.Y.

\begin{tabular}{|l|l|l|l|l|l|l|l|}
\hline Table 1. Summarization of the characteristics of the cases \\
\hline & $\begin{array}{l}\text { Age } \\
\text { (year) }\end{array}$ & Sex & Number of lesions & Size (mm) & Approach & $\begin{array}{l}\text { Follow-up } \\
\text { (months) }\end{array}$ & $\begin{array}{l}\text { Change in e-GFR } \\
\text { in follow-ups (\%) }\end{array}$ \\
\hline Case 1 & 45 & F & 1 & $60 \times 50$ & LPN & 11 & -19.1 \\
\hline Case 2 & 58 & M & 1 & $80 \times 70$ & LRN & 67 & -32.5 \\
\hline Case 3 & 62 & $\mathrm{M}$ & 2 & $38 \times 30$ and 10x7 & LRN & 47 & -55.4 \\
\hline Case 4 & 89 & F & 2 & 40 and 30 & LRN & 14 & -24.1 \\
\hline LPN: Laparoscopic partial nephrectomy, LRN: Laparoscopic radical nephrectomy, mm: Millimeter, F: Female, M: Male \\
\hline
\end{tabular}




\section{Acknowledgements}

Publication: The results of the study were not published in full or in part in form of abstracts.

Contribution: There is not any other contributors who may not be listed as authors.

Conflict of Interest: No conflict of interest was declared by the authors.

Financial Disclosure: The authors declared that this study received no financial support.

\section{References}

1. Larbcharoensub N, Limprasert V, Pangpunyakulchai D, et al. Renal Leiomyoma: A Case Report and Review of the Literature. Urol Case Rep 2017;13:3-5.

2. Ghadian A, Hoseini SY. Transvesical enucleation of multiple leiomyoma of bladder and urethra. Nephrourol Mon 2013;5:709-711.

3. Gupta A, Chandra N, Sharma A, et al. Renal leiomyoma in a child: a rare renal tumor. J Pediatr Surg 2010;45:1900-1903.
4. Romano FU, Gonzalez-Serrano A, Moreno-Aranda J. Renal Pelvis Leiomyoma- An Infrequent Clinical Case. Journal of clinical and diagnostic research: JCDR 2017;11:PD12-PD13.

5. Mitra B, Debnath S, Pal M, et al. Leiomyoma of kidney: An Indian experience with literature review. Int J Surg Case Rep 2012;3:569-573.

6. Khetrapal S, Bhargava A, Jetley $S$, et al. Renal leiomyoma: an uncommon differential diagnosis of renal masses with a clinical relevance. J Clin Diagn Res 2014;8:FD08-9.

7. Lee SY, Hsu HH, Chang CT, et al. Renal capsular leiomyoma--imaging features on computed tomography and angiography. Nephrol Dial Transplant 2006;21:228-9.

8. Izadpanahi $\mathrm{MH}$, Kabiri M, Mazdak $\mathrm{H}$, et al. Preoperative evaluation of pelvic kidney renal cell carcinoma with 64-slice CT and 3D-CT angiography. Adv Biomed Res 2014;3:250.

9. Sivrioglu AK, Tutar $S$, Kafadar $C$, et al. The diffusion-weighted imaging of renal leiomyoma. Abdom Radiol (NY) 2016;41:1215-1216.

10. Kolaric V, Ajdukovic D, Racz A. Nursing evaluation of diabetes selfmanagement in tertiary healthcare settings in Croatia. Psychiatr Danub 2014;26 Suppl 3:513-519.

11. Nishiyama R, Kanatani I, Oka H, Ichijima K. [Laparoscopic partial nephrectomy for renal leiomyoma: a case report]. Hinyokika Kiyo 2012;58:197-201. 